\title{
Health technology in school-based health centers: supporting continuous care during COVID-19
}

\author{
Erin Sullivan $^{1}$, Anna Goddard ${ }^{2}$, Paula Fields ${ }^{1}$, Suzanne Mackey ${ }^{1}$ \\ ${ }^{1}$ The School-Based Health Alliance, Washington, DC, USA; ${ }^{2}$ Sacred Heart University, Fairfield, CT, USA \\ Contributions: (I) Conception and design: All authors; (II) Administrative support: All authors; (III) Provision of study materials or patients: P Fields, \\ E Sullivan; (IV) Collection and assembly of data: P Fields, E Sullivan; (V) Data analysis and interpretation: E Sullivan; (VI) Manuscript writing: All \\ authors; (VII) Final approval of manuscript: All authors. \\ Correspondence to: Erin Sullivan. 1010 Vermont Ave NW \#816, Washington, DC 20005, USA. Email: esullie@gmail.com.
}

\begin{abstract}
Background: Children and adolescents in the United States face disparities by race, ethnicity, and socioeconomic status, including unequal access to consistent and high-quality healthcare. School-based health centers (SBHCs) promote health equity by delivering primary, mental, and other health services directly to students in their schools. The COVID-19 outbreak in the United States resulted in schools and SBHCs closing their physical sites and pivoting to virtual service delivery.

Methods: In the months immediately following school closures, school-based health center practitioners and sponsors participated in an online listening series to share how they used technology to creatively advertise services, engage with students, and continue delivering care. These discussions highlight challenges, opportunities, and future implications for telehealth and the use of technology in SBHCs.

Results: With no or limited opportunity to interact with patients in-person, school-based health center staff used technology to conduct marketing and outreach and offer virtual care. Many launched telehealth operations for the first time due to the pandemic, whereas others altered or expanded previously established virtual service offerings. Listening session participants recognize the unique benefits of telehealth during and after the pandemic, but they also discussed unique challenges, such as privacy concerns and the digital divide. Conclusions: The findings outlined in this manuscript should serve as a baseline for future research and programs related to school-based health, health technology, and pediatric care, especially during global crises. Health technology, particularly telehealth, is a crucial tool to mitigate strain on emergency departments, administer screening and preventative care, and provide mental health care as COVID cases continue to rise. Enabling policy solutions, shared best practices, and supportive partnerships are crucial as SBHCs continue to find ways to help students remain healthy and achieve their fullest potential.
\end{abstract}

Keywords: Telehealth; school-based healthcare; COVID-19

Received: 30 October 2020; Accepted: 21 May 2021; Published: 30 November 2021. doi: $10.21037 /$ ht-20-30

View this article at: http://dx.doi.org/10.21037/ht-20-30

\section{Introduction}

Disparities in the United States (U.S.) by race and socioeconomic status are well-documented (1-4). Youth in low-income communities have higher rates of asthma, obesity, and depression (5), and those of racial and ethnic minority groups are more likely to develop chronic health problems and less likely to have a regular source of medical care (1). More than $25 \%$ of children in the U.S. face access barriers to essential health care-including lack of transportation, low health literacy, and provider shortagesand a similar amount have unmet needs for specialty care (6). There are 1.5 million U.S. households that do not own a car, $40 \%$ of rural communities that lack public transportation services, and 14 million children who live in areas with health professional shortages (6). 
Table 1 SBHC Delivery Model Definitions and Proportions (10)

\begin{tabular}{lllll}
\hline Delivery model & Traditional & School-linked & Mobile & Telehealth exclusive \\
\hline $\begin{array}{l}\text { Location where patient } \\
\text { access care }\end{array}$ & $\begin{array}{l}\text { A fixed site on a school } \\
\text { campus }\end{array}$ & $\begin{array}{l}\text { A fixed site near a } \\
\text { school campus }\end{array}$ & $\begin{array}{l}\text { A mobile van parked on } \\
\text { or near a school campus }\end{array}$ & $\begin{array}{l}\text { A fixed site on a } \\
\text { school campus }\end{array}$ \\
$\begin{array}{l}\text { Location where primary care } \\
\text { providers are delivering care }\end{array}$ & $\begin{array}{l}\text { Physically on-site at the } \\
\text { SBHC }\end{array}$ & $\begin{array}{l}\text { Physically on-site at the } \\
\text { SBHC }\end{array}$ & $\begin{array}{l}\text { Physically on-site at the } \\
\text { Remotely }\end{array}$ \\
$\begin{array}{l}\text { Proportion of SBHCs } \\
\text { nationwide }\end{array}$ & 82 & 4 & 3 & 12 \\
\hline
\end{tabular}

SBHC, School-based health center.

School-based health centers (SBHCs) are an evidencebased solution for achieving health equity among youth (7), recognized for their significant positive effects on high school completion, hospital and emergency room admittance, substance abuse, and contraception use (8). SBHCs improve preventative service delivery, decrease asthma morbidity, increase mental and behavioral health access and utilization (7) and, in turn, increase students' time spent learning $(7,9)$. SBHCs represent partnerships between schools and community health organizations and demonstrate a shared commitment to providing student health access in a safe, convenient location. In a majority $(82 \%)$ of SBHCs, students access care in person at a fixed site on a school campus. In the remainder, students access primary care in person at a fixed site near a school campus (4\%), in person on a specially equipped van or bus parked on or near a school campus (3\%), or at a fixed site on a school campus through telehealth (12\%; Table 1) (10). SBHCs employ a minimum of one primary care practitioner, but many offer a multidisciplinary team of medical, mental, oral, and vision providers (11). These practitioners provide services to students from pre-kindergarten through high school, and sometimes to school staff, students' family members, and the community (10).

Across the U.S., 2,584 SBHCs provide more than 6.3 million students access to care (10). An average of $70 \%$ of students in schools with SBHC access are eligible for free or reduced-price lunch, compared to $55 \%$ of students in all public schools in the U.S (10). In recent years, new SBHCs have been opening more rapidly in rural and suburban areas than in urban settings, aiming to address the transportation and provider shortages that are significant barriers to care (12). As the number of SBHCs in rural areas grows, so does the number of those offering telehealth services. In 2016, the one in five centers that used telehealth to provide services were more likely to serve rural communities $(10,13)$.

When widespread COVID-19 transmission began in the U.S. in spring 2020, health systems had to find creative, immediate solutions to continue serving patients through limited social contact. This affirmed the importance of telehealth, a modality that presents myriad benefits including convenience, cost reduction, and infection control $(14,15)$. However, the pandemic and related restrictions also unearthed challenges in launching and maintaining telehealth, including practitioner acceptance and buy-in, reimbursement policies, organizational readiness, and a digital divide (14).

When schools around the U.S. closed in spring 2020 due to the pandemic, preliminary results from a national SchoolBased Health Alliance (the Alliance) survey indicated that as many as three quarters of SBHC physical sites closed temporarily, and over half of SBHCs used telehealth to provide services to students (16). This paper describes the innovative ways SBHCs used health technology, including telehealth, to conduct outreach and provide care to highneed students in the months following COVID-related school closures. Specifically, qualitative data and analyses investigate three key research questions: (I) changes in SBHC outreach and communications due to COVID-19 and school closures, (II) changes in service delivery due to COVID-19 and school closures, and (III) supports and challenges to continuity of care at SBHCs throughout the COVID-19 pandemic. These findings outline barriers and facilitators, success stories, and implications for policy and practice that should serve as a baseline for future research and programs related to school-based health, health technology, and pediatric care. 
Table 2 Listening Session Registered and Attending Participants

\begin{tabular}{lcccc}
\hline Participant type & Session date & Session topic & Total registered & Total attended \\
\hline SBHCs & April 30, 2020 & Telehealth & 202 & 113 \\
& May 7, 2020 & Re-entry & 143 & 77 \\
& May 21, 2020 & Mental health & 178 & 62 \\
SBHC Sponsor & April 28, 2020 & Telehealth & 182 & 50 \\
Organizations & May 5, 2020 & Re-entry & 101 & 50 \\
& May 19, 2020 & Mental health & 112 & 48 \\
\hline
\end{tabular}

SBHC, School-based health center.

\section{Methods}

\section{Recruitment}

To support SBHCs during unprecedented times, the Alliance organized six free 60- to 90-minute online discussions for SBHC and SBHC sponsor organization staff to share strategies and challenges related to operations during the pandemic. The Alliance maintains a database with contact information of the 2,584 SBHCs nationwide, described in detail elsewhere (10). Before each discussion, the Alliance emailed event and registration to all database contacts. Each discussion was limited to 150 participants due to restrictions of the host platform (17).

\section{Procedure}

Three of the discussions included only SBHC staff and the remaining three included only SBHC sponsor organization staff. Each of the three sessions for each participant group focused on a topic related COVID-19 SBHC operations: telehealth, mental health, and re-entry into the 2020-21 school year. At the beginning of each discussion, hosted through Zoom (17), the Alliance moderator disclosed that it would be recorded, transcribed, and shared. The moderator followed a semi-structured guide, and participants responded to each question in writing through Menti (18), an interactive online platform that displayed real-time aggregate responses through data visualizations. Two to three content experts from the Alliance joined each discussion to monitor the written discussion and flag themes and innovations to the moderator. The moderator then read these select responses aloud and called on the writer to elaborate.

\section{Data analysis}

Each discussion audio recording was transcribed by Zoom. Menti responses, Zoom chat box text responses, and Zoomgenerated audio transcripts were extracted and downloaded after each discussion. Participant data, specifically state, organization name, and professional role, were analyzed and presented in table form (Table 2). To maintain anonymity, researchers de-identified participants.

Two Alliance researchers analyzed the transcribed text, chat box text, and Menti text using qualitative content analysis (19). The researchers first read the transcripts and written text repeatedly to achieve familiarization and immersion. Responses were then categorized by research questions: (I) changes in SBHC outreach and communications due to COVID-19 and school closures, (II) changes in service delivery due to COVID-19 and school closures, and (III) supports and challenges to continuity of care at SBHCs throughout the pandemic. Within each category, the researchers identified and coded common themes. The researchers employed a constant comparison technique to develop consistent themes and form agreement. Additionally, the study team invited a thirdparty researcher from Sacred Heart University to analyze detailed audit trails, critique study methods and conclusions, and limit researcher bias. Reports detailing aggregate and de-identified findings served as the basis for this manuscript. This research focused on organizational practices and did not require Institutional Review Board review.

\section{Participants}

The number of participants per session ranged from 48 to 113, with an average of 72 (Table 2). Most describe their occupations as SBHC directors, SBHC administrators, 
social workers, behavioral health clinicians, and primary care clinicians. Those participating represent 41 states and are evenly dispersed by geographic region, with a slight majority in the U.S. South and West (20). This parallels geographic spread of SBHCs nationally (10). However, representatives from eight states with identified SBHCs (IA, ID, MI, NE, NH, NV, UT, and V'T) did not choose to participate.

\section{Results}

\section{Virtual outreach, marketing, and communications}

Without the opportunity to interact with students in person, SBHCs conducted outreach proactively using technology. Some used emails, social media, and text messages to remind clients about scheduled appointments or connect caregivers with mental health, food assistance, and technology support. Staff at one SBHC called students and their families to help them activate their telehealth portal accounts, increasing the activation rate from $5 \%$ to $37 \%$ in just one week. SBHCs also targeted outreach to specific sub-populations, such as asthmatics and behavioral health patients. One site scanned enrollment forms for students with documented health issues and then conducted courtesy calls explaining how to schedule telehealth visits.

SBHCs used technology to disseminate health information and advertise services. One created bi-weekly newsletters that school partners shared on school social media platforms, and others engaged with students through the Google Classrooms established by schools. A participant mentioned sharing lighthearted videos with self-care tips as a way to interact with students who may not schedule formal visits. Several noted more actively participating in social media or creating healthcare hotlines. One SBHC program developed a website for students to access crisis information, health education, and counselors. The site also hosted a virtual meeting every day for students to discuss health and wellness topics.

\section{Virtual care}

SBHCs altered service delivery in response to the changing landscape. Many conducted virtual screenings of patients for COVID symptoms by phone before in-person care or had brief phone check-ins to assess urgent needs and arrange follow-ups as indicated. Some SBHCs conducted risk assessments through telehealth and the remainder of a visit in person. SBHCs advocated using telehealth to increase check-ins with high-risk patients.

Technology played a crucial role in virtual physical care appointments. Providers collected patient-generated vital signs through phone applications, assessed range of motion and skin through phone cameras, and watched patients jump on video to evaluate abdominal pain. Providers described ways in which they involved parents for handson assistance during care delivery, such as using a flashlight to look into the throat during dental visits or pressing down on the stomach to assess abdominal pain. SBHCs expressed a heightened need to collaborate and stay in closer contact with caregivers when in-person care is not available.

To administer services, participants reported use of various services, including Doxy.me, Zoom, swyMed, FaceTime, Google Classroom, Skype, Zoom, Google Voice, phone calls, and text messaging systems. Provider and patient resources, in combination with state and sponsor policies, determined the platforms used.

\section{Telehealth expansion}

SBHCs that provided telehealth before the COVID-19 outbreak expanded or adjusted their models in response to the pandemic. For instance, some offered telehealth services to the entire community when they had previously only served students. Others expanded telehealth services to include acute care or focus on mental health visits. One SBHC collaborated with an in-person pediatric and adolescent obesity specialty clinic to launch telehealth to rural student populations for access to these services when SBHCs reopened. Another provided "senior transition visits" via telehealth, reviewing each graduating senior's history and medical record to ensure they would be ready to transition care.

\section{Opportunities and challenges}

SBHCs recognized telehealth as a crucial strategy to deliver care in the 2020-21 school year. Several participants discussed using telehealth to supplement in-person care and limit the number of clients visiting the health center in person. Some saw telehealth as a tool to extend care beyond the typical school day or school year or as a platform to care for students absent from school. Telehealth could also provide a pathway to maintain connections with graduating seniors. Instead of transitioning these students to adult care, clinicians could continue to provide virtual "young adult" 
care. Practitioners remarked that telehealth offers a unique opportunity to view patients at home, providing a more holistic view of the patient and environment than a typical office visit.

These opportunities present unique challenges, including privacy and confidentiality concerns. Participants acknowledged that in both individual and group sessions, students were not always comfortable sharing a view into a space that is usually private. Some students preferred only to show their faces at the beginning of the session and then direct the camera to the ceiling, while others completed sessions from spaces like closets for added privacy. To ensure privacy during a visit, providers suggested that students take a walk, make calls from a parked car, or find other ways to distance themselves from others. Providers recognized that patients might not find an isolated space, so they took extra steps to ensure confidentiality during visits. For example, providers confirmed if anyone other than the patient was present at the beginning of the session or conducted the visit in writing through the chat function on the telehealth platform. For those completing visits with visual capabilities, participants recommended starting the appointment with clinicians showing their space and then asking patients to do the same to establish a sense of security. Instead of collecting one general contact number, SBHCs designated between the student and caregiver cell phone number in the electronic health record. SBHCs could therefore text and call students directly to notify them about the availability of confidential visits.

\section{Discussion}

While technology has proven to be an invaluable tool for providing health care to children and adolescents throughout the COVID-19 pandemic, there are challenges to establish and maintain virtual services. For one, telehealth equipment can require a considerable monetary investment, which may deter resource-limited health centers (21). Under normal circumstances, when providers cannot use a variety of technology platforms or deliver care to patients directly in their homes, telehealth implementation requires physical space, staffing, technology, and partnerships (22). These barriers may seem insurmountable for health centers that barely have the bandwidth to serve students in person.

The reliance on technology during the COVID-19 outbreak has shined a light on the digital divide across the U.S. About a third of households with children ages 6 to 17 with an annual income less than $\$ 30,000$ lack high- speed internet connection at home, and 25\% lack computer access at home (23). This divide widens in rural areas, where telehealth is crucial for overcoming barriers like lack of transportation and provider shortages. Only $69.3 \%$ of rural areas have access to high-speed broadband internet that meet the minimum benchmark set by the Federal Communications Commission (22). This impedes patients from participating in video consultations with providers and using technology to monitor their health at home.

Before the COVID-19 outbreak, laws and policies created additional challenges for SBHCs hoping to establish or expand telehealth. State Medicaid agencies define telehealth parameters, including the types of authorized providers, reimbursable services, acceptable technology platforms, and permitted locations for patients during care. This is difficult for practitioners to navigate when providing care in multiple states (24). States typically require providers to be licensed in the state where the patient receives services, but pursuing licensure in different states can be time-intensive and costly (22). Some states limit the types of providers that can provide services through telehealth, and these lists tend to exclude federally qualified health centers (FQHCs), the sponsor organization type of a majority of SBHCs (24).

Temporary policy expansions allowed providers to use various technology platforms to deliver care and conduct virtual visits to patients. Under normal circumstances, platforms like email and telephone are rarely acceptable forms of delivery in terms of Medicaid reimbursement, unless in conjunction with another acceptable system $(24,25)$, and Medicaid programs typically exclude the home as a reimbursable site. Only ten state Medicaid programs explicitly allow the home to serve as an originating site, but there are many additional restrictions and a facility fee is not billable $(14,24)$.

The Centers for Medicare and Medicaid Services (CMS) provided state Medicaid programs with increased authority and flexibility to expand telehealth services during the pandemic, such allowing telephonic care, removing crossstate licensing requirements, and allowing FQHCs to provide telehealth services as distant site providers $(26,27)$. State decisions on specific policies vary. While CMS stated their actions will be temporary to address the COVID pandemic, this increased state authority and flexibility must be permanent to address this crisis's long-term impacts.

SBHCs need increased state and federal funding to maintain services. Many states provide direct and indirect 
funding to SBHCs, which is critical to sustainability. As states face significant budget shortfalls related to the pandemic, they must maintain their investment in SBHCs. While some SBHCs receive federal funds through various programs, there is no dedicated federal funding program for SBHCs. Future federal COVID relief legislation must prioritize support for SBHCs.

Although telehealth service delivery dramatically increased in recent months, it has not outpaced the decline in primary, preventative, and mental health service administration among the populations who need these services most (28). While many schools remain closed, essential health services like screenings and vaccinations are further delayed, presenting significant short- and long-term effects on health outcomes. Compared to the same months in 2019, between March and May 2020, there were 44\% fewer outpatient mental health services, $44 \%$ fewer physical and cognitive development screenings, and $69 \%$ fewer dental services administered (28). This gap in healthcare delivery translates to lifelong consequences for children and adolescents.

The use and advancements of health technology, particularly in times of crisis, have allowed practitioners to deliver care to those who may not otherwise receive it. The COVID-19 outbreak in the U.S. led to incredible innovation, but it also highlighted widespread disparities and challenges. Enabling policy solutions, increased funding, and supportive partnerships are crucial as SBHCs continue to use technology to help students remain healthy and achieve their fullest potential.

\section{Limitations}

The authors recognize several limitations. The large group setting could have thwarted participation, and the format may have encouraged only those successfully delivering services or modifying service delivery to share. Since the Alliance advertised the sessions as a space for respondents to listen, some may have joined only to learn from others. Similarly, information shared was selfreported by participants and the researchers did not verify the information reported. The opt-in convenience sample may not represent of the field at-large nor include representatives from closed or resource-limited sites. Finally, the researchers' pre-existing knowledge may have influenced their analyses and the resulting themes and topics, although independent review and subsequent consensus building aimed to mitigate this.

\section{Acknowledgments}

The authors would like to acknowledge the SchoolBased Health Alliance for organizing and implementing the discussion forum referenced in this manuscript. In particular, School-Based Health Alliance staff Katherine Cushing assisted in analyzing the information collected, and Andrea Shore moderated the discussion forum. Laura Brey and Matthew Even of the Alliance and Samira Soleimanpour of the University of California, San Francisco led the implementation of the COVID-related survey of SBHCs referenced throughout the manuscript. Finally, the authors would like to acknowledge the school-based health center staff and sponsor organization members who participated in the Alliance-led discussion forum and shared their innovations in care delivery.

Funding: None.

\section{Footnote}

Data Sharing Statement: Available at http://dx.doi. org/10.21037/ht-20-30

Conflicts of Interest: All authors have completed the ICMJE uniform disclosure form (available at http://dx.doi. org/10.21037/ht-20-30). ES serves as an unpaid editorial board member of Health Technology from June 2020 to May 2022. The other authors have no conflicts of interest to declare.

Etbical Statement: The authors are accountable for all aspects of the work in ensuring that questions related to the accuracy or integrity of any part of the work are appropriately investigated and resolved. Reports describing aggregate and de-identified findings served as the basis for this manuscript. This project did not qualify for human subjects' research and did not require Institutional Review Board review.

Open Access Statement: This is an Open Access article distributed in accordance with the Creative Commons Attribution-NonCommercial-NoDerivs 4.0 International License (CC BY-NC-ND 4.0), which permits the noncommercial replication and distribution of the article with the strict proviso that no changes or edits are made and the original work is properly cited (including links to both the formal publication through the relevant DOI and the license). See: https://creativecommons.org/licenses/by-nc-nd/4.0/. 


\section{References}

1. Bloom B, Cohen RA, Freeman G. Summary health statistics for u.s. Children: national health interview survey, 2011. Vital Health Stat 10. 2012;(254):1-88.

2. Centers for Disease Control and Prevention. Health disparities and inequalities report: United States. 2011 [cited 2021 March 29]. Available online: https://www. cdc.gov/minorityhealth/chdir/2011/CHDIR2011. html\#anchor_1606326829

3. Kann L, Kinchen S, Shanklin SL, et al. Youth risk behavior surveillance--United States, 2013. MMWR Suppl 2014;63:1-168. Erratum in: MMWR Morb Wkly Rep. 2014;63:576.

4. Agency for Healthcare Research and Quality. 2012 National healthcare quality report. Rockville, Maryland: 2013 [cited 2021 March 29]. Available online: https://www. ahrq.gov/sites/default/files/publications/files/2012nhqr.pdf

5. Irwin CE Jr, Adams SH, Park MJ, et al. Preventive care for adolescents: few get visits and fewer get services. Pediatrics. 2009;123:e565-72.

6. Redlener I, Gracy D, Walto D, et al. More than 20 million children in U.S. still lack sufficient access to essential health care. Children's Health Fund; 2016 [cited 2021 March 29]. Available online: https://www. childrenshealthfund.org/wp-content/uploads/2016/11/ Unfinished-Business-Final_.pdf

7. Knopf JA, Finnie RK, Peng Y, et al. School-based health centers to advance health equity: a community guide systematic review. Am J Prev Med 2016;51:114-26.

8. Guide to Community Preventive Services. Health equity: school-based health centers. [website]. Available online: https://www.thecommunityguide.org/findings/promotinghealth-equity-through-education-programs-and-policiesschool-based-health-centers

9. Keeton V, Soleimanpour S, Brindis CD. School-based health centers in an era of health care reform: building on history. Curr Probl Pediatr Adolesc Health Care 2012;42:132-56; discussion 157-8.

10. Love HE, Schlitt J, Soleimanpour S, et al. Twenty years of school-based health care growth and expansion. Health Aff (Millwood) 2019;38:755-64.

11. School-Based Health Alliance [website]. Available online: www.sbh4all.org

12. Love H, Soleimanpour S, Panchal N, et al. 201617 National school-based health care census report.
Washington: School-Based Health Alliance, 2018.

13. Love H, Panchal N, Schlitt J, et al. The Use of Telehealth in School-Based Health Centers. Glob Pediatr Health 2019;6:2333794X19884194.

14. Smith AC, Thomas E, Snoswell CL, et al. Telehealth for global emergencies: Implications for coronavirus disease 2019 (COVID-19). J Telemed Telecare 2020;26:309-13.

15. Wijesooriya NR, Mishra V, Brand PLP, et al. COVID-19 and telehealth, education, and research adaptations. Paediatr Respir Rev 2020;35:38-42.

16. COVID-focused survey of School-Based Health Centers. Washington, DC: The School-Based Health Alliance; 2020.

17. Zoom. San Jose, CA: Zoom Video Communications, Inc.; 2021.

18. Mentimeter. Stockholm, Sweden: Mentimeter AB; 2021.

19. Graneheim UH, Lundman B. Qualitative content analysis in nursing research: concepts, procedures and measures to achieve trustworthiness. Nurse Educ Today. 2004;24:105-12.

20. Census Regions and Divisions of the United States. Washington, DC: United States Census Bureau; 2010.

21. Telemedicine in school-based health centers: a profile of the center for rural health innovation. American Public Health Association; 2018.

22. Rocha L, Powers M, Siegfried A. Rural telehealth toolkit. NORC Walsh Center for Rural Health Analysis; 2019.

23. Auxier B, Anderson M. As schools close due to the coronavirus, some U.S. students face a digital 'homework gap'. 2020. Available online: https://www.pewresearch. org/fact-tank/2020/03/16/as-schools-close-due-tothe-coronavirus-some-u-s-students-face-a-digitalhomework-gap/?utm_content=buffer09ef5\&utm_ medium=social\&utm_source=twitter.com\&utm_ campaign=buffer

24. Kwong M. State telehealth laws and reimbursement policies: a comprehensive scan of the 50 states and district of Columbia. Sacramento, CA: Center for Connected Health Policy; 2018.

25. Verma S. Early impact of CMS expansion of Medicare telehealth during COVID-19. Health Affairs 2020. Available online: https://www.healthaffairs.org/ do/10.1377/hblog20200715.454789/abs/

26. U.S. Department of Health \& Human Services. Telehealth: delivering care safely during COVID-19. 2021. Available online: https://www.hhs.gov/coronavirus/ 
telehealth/index.html

27. Robeznieks A. Key changes made to telehealth guidelines to boost COVID-19 care. American Medical Association (AMA). 2020.

\section{doi: $10.21037 /$ ht-20-30}

Cite this article as: Sullivan E, Goddard A, Fields P, Mackey S. Health technology in school-based health centers: supporting continuous care during COVID-19. Health Technol 2021;5:11.
28. CMS issues urgent call to action following drastic decline in care for children in Medicaid and Children's Health Insurance Program due to COVID-19 pandemic [press release]. Centers for Medicare \& Medicaid Services. 2020. 